\begin{tabular}{|c|l|}
\hline Title & A coustic whispering-gall lery modes generated and dynamically imaged with ultrashort optical pul ses \\
\hline Author(s) & Tachizaki, Takehiro; Matsuda, Osamu; Maznev, A lex A.; Wright, Oliver B. \\
\hline Citation & $\begin{array}{l}\text { Physical Review B, 81(16), 165434 } \\
\text { https://doi.org/10.1103/PhysRevB.81.165434 }\end{array}$ \\
\hline Issue Date & 2010-04_15 \\
\hline Doc URL & http://hdl.handle.net/2115/43062 \\
\hline Rights & @2010 The A merican Physical Society \\
\hline Type & article \\
\hline File Information & PRB81-16_165434.pdf \\
\hline
\end{tabular}

Instructions for use 


\title{
Acoustic whispering-gallery modes generated and dynamically imaged with ultrashort optical pulses
}

\author{
Takehiro Tachizaki, Osamu Matsuda, Alex A. Maznev, and Oliver B. Wright* \\ Division of Applied Physics, Graduate School of Engineering, Hokkaido University, Sapporo 060-8628, Japan
}

(Received 27 February 2010; published 23 April 2010)

\begin{abstract}
Broadband coherent surface phonon wave packets up to $1 \mathrm{GHz}$ in a microscopic embedded disk are excited and dynamically imaged with ultrashort optical pulses. We isolate a whispering-gallery-like mode using a technique involving quadrature detection of the out-of-plane surface motion on modulating the optical excitation beam. The eigenmode distribution is obtained from spatiotemporal Fourier transforms and a radialazimuthal decomposition demonstrates the selective excitation of a mode with 26-fold rotational symmetry and allows an estimate of the cavity $Q$ factor to be made.
\end{abstract}

DOI: 10.1103/PhysRevB.81.165434

PACS number(s): 63.20.Pw, 43.35.+d, 63.20.D-, 78.20.hc

\section{INTRODUCTION}

Since Rayleigh's discovery of acoustic whispering gallery (WG) modes propagating in the air around the dome of St. Paul's Cathedral 100 years ago, ${ }^{1}$ a variety of such modes have been investigated. It is now recognized that sound waves in solid or liquid spheres, cylinders, or disks can also be trapped as WG modes, ${ }^{2-5}$ of significance in the field of nondestructive testing of bearings or pipes, in gas sensing, and in signal filtering for telecommunications. ${ }^{6-10}$ Confined phonon fields such as WG or microcavity modes are of great interest because of their potential for strong acousto-optic interactions or cavity optomechanics. ${ }^{11-13}$ Electromagnetic WG modes in the optical, infrared, or microwave regions in spheres, disks, or toroids, ${ }^{14-19}$ as well as recently demonstrated plasmon- and exciton-polariton WG modes, ${ }^{20,21}$ also have numerous applications such as in microlasing or in single-molecule sensing.

WG resonators are often studied in the spectral domain. It has also proved possible to spatially image acoustic, optical, or microwave WG modes. ${ }^{18,22,23}$ The most complete means of investigating WG modes is by real-time imaging. However, in spite of the enormous interest in this field, the only such measurements to date are on optical WG modes, but without sufficient temporal resolution for spectral analysis. ${ }^{24}$

Here we implement an ultrafast interferometric technique 25,26 to dynamically image optically induced surface phonons on a microscopic copper disk embedded in silica on a Si substrate. We thereby temporally track the evolution of the amplitude and phase of an acoustic WG-like mode in two dimensions (2D) and access its dispersion. The $Q$ factor is estimated by means of a quadrature detection of the surface motion on modulating the optical excitation beam.

\section{SAMPLE AND APPARATUS}

The disk resonator, manufactured by International Sematech, consists of a polycrystalline copper disk of diameter $37.5 \mu \mathrm{m}$ embedded in a silicon oxide $\left(\mathrm{SiO}_{2}\right)$ layer of thickness $370 \mathrm{~nm}$ [see Figs. 1(a) and 1(b)]. The fabrication, based on a damascene process, involves patterning the dielectric film, depositing a $25 \mathrm{~nm}$ Ta diffusion barrier between the $\mathrm{Cu}$ and $\mathrm{SiO}_{2}$ (not shown) and a seed $\mathrm{Cu}$ layer by physical vapor deposition, depositing the bulk of $\mathrm{Cu}$ by electroplating, and, finally, polishing off the excess $\mathrm{Cu}$ by chemical-mechanical polishing (CMP). The $\mathrm{Cu}$ is overpolished and recessed with respect to the surface of the dielectric by up to $\sim 150 \mathrm{~nm}$. This phenomenon called "dishing" is evident in atomic force microscope (AFM) profiles measured across the disk diameter in Fig. 1(c). This structure lies on a $100 \mathrm{~nm}$ silicon nitride and a $550 \mathrm{~nm} \mathrm{SiO}_{2}$ layer, all resting on a singlecrystal (100) silicon substrate. The anisotropy of $\mathrm{Si}$ is relatively weak ${ }^{27}$ and, moreover, the isotropic film layer is relatively thick, so the presence of $\mathrm{Si}$ does not significantly affect the symmetry of the observed wave fields.
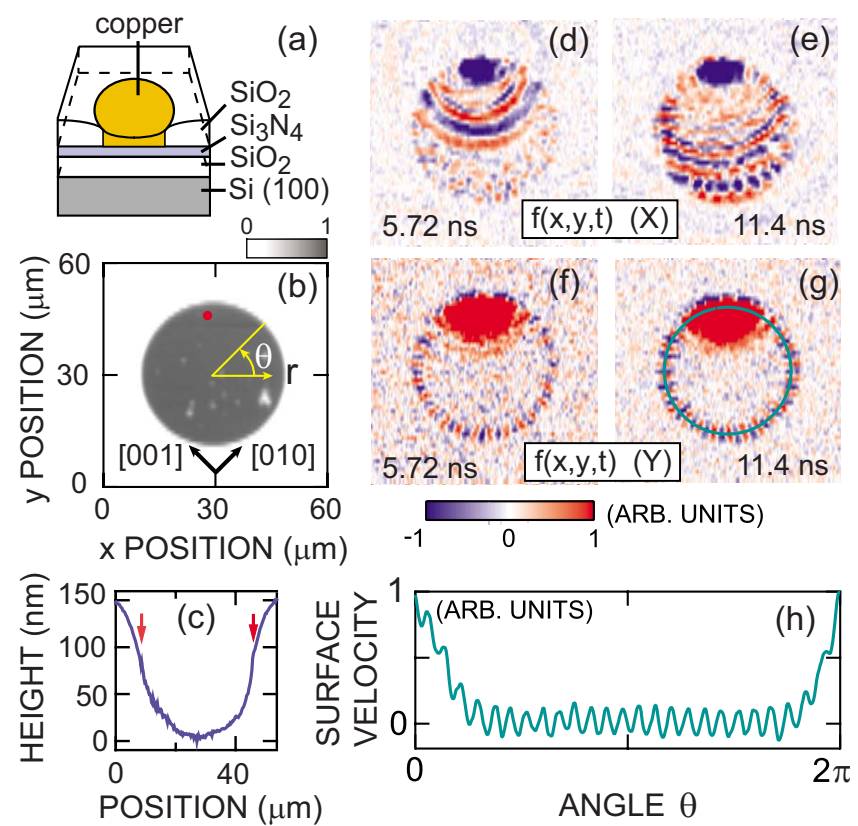

FIG. 1. (Color online) (a) Sample structure and section. (b) Normalized probe reflectivity map. Dot shows the pumping position. (c) AFM profile across the disk diameter. Arrows: edge of disk. (d), (e) and (f), (g) are surface phonon images of the in-phase $(X)$ and the quadrature $(Y)$ components of $\Delta \phi$ at fixed delay times 5.72 and 11.40 ns after pump pulse arrival, respectively. (h) Profile of the quadrature component along the circle in (g) (counterclockwise from the top). 
Broadband surface acoustic waves with frequencies up to $1 \mathrm{GHz}$ are generated thermoelastically at a point $\sim 3 \mu \mathrm{m}$ from the edge of the copper disk [see normalized probe reflectivity image of Fig. 1(b)] by normally incident ultrashort pump light pulses of central wavelength $405 \mathrm{~nm}$, a repetition rate of $76 \mathrm{MHz}$, a duration of $\sim 200 \mathrm{fs}$, and a pulse energy of $0.1 \mathrm{~nJ}$. The pump beam, focused by a $\times 50$ microscope objective of numerical aperture 0.45 and working distance 15 $\mathrm{mm}$ to a $\sim 1 \mu \mathrm{m}$ radius spot, is modulated acousto-optically at $1.1 \mathrm{MHz}$ for lock-in detection. Time-delayed normally incident synchronous probe pulse pairs at an interval of $500 \mathrm{ps,}$ a wavelength of $810 \mathrm{~nm}$, and a pulse energy of $0.01 \mathrm{~nJ}$ are focused with the same objective to a similar spot size to detect the out-of-plane surface velocity $(\sim 10 \mathrm{pm}$ in amplitude) through the optical phase difference $\Delta \phi \sim 10^{-4}$ in a Sagnac interferometer. ${ }^{26}$ We obtain images with $\sim 2 \mu \mathrm{m}$ lateral resolution with a system based on a tilting mirror and a confocal lens pair that allows the probe spot to be raster scanned in two dimensions relative to that of the pump. By incrementing the delay time between the pump and probe, we build up an animation ${ }^{28}$ of 30 frames over the laser repetition period of $13.2 \mathrm{~ns}$ with a temporal resolution, $\sim 1 \mathrm{ps}$, determined by probe beam dispersion in the optical delay line. The acquisition time for one frame is approximately 5 $\min$.

\section{RESULTS}

\section{A. In-phase and quadrature real-time data}

Examples of surface phonon images for a $60 \times 60 \mu \mathrm{m}^{2}$ region encompassing the copper disk are shown in Figs. 1(d) and 1(e). These data are obtained, as is standard practice, from the in-phase (termed $X$ ) output of the lock-in amplifier at delay times 5.72 and $11.40 \mathrm{~ns}$, respectively, where inphase means with respect to the pump beam modulation envelope. Nonlocalized surface acoustic waves (referred to here simply as SAWs) emanating from the excitation point are evident in these images over the $\mathrm{Cu}$, where the probe reflectivity is significant, although SAWs in the region outside the disk in Fig. 1(d) and 1(e) are also faintly visible. The petal-like structure at the bottom of Fig. 1(e) suggests the presence of a localized SAW WG mode guided around the disk circumference, but the SAWs prevent this mode from being properly resolved. However, when the quadrature output (termed $Y$ ) — a component that is normally discarded-is imaged, as shown in Figs. 1(f) and 1(g) for same time delays, the SAWs vanish and a WG-like mode appears. A cross section of the WG-like mode (hereafter referred to as a WG mode for brevity) in Fig. 1(g), shown in Fig. 1(h), exhibits a sinusoidal variation in the regions outside the heated pump spot. The moment of SAW generation, the coupling of the SAWs to the WG mode, and the WG mode time evolution are best viewed in the real-time animations of the $X$ and $Y$ components. $^{28}$

These results can be qualitatively understood by considering the lifetime of the WG mode: since the traveling waves making up this mode propagate round the disk, this mode is expected to be long-lived compared to the time a given SAW wave front is imaged. This results in a delayed response and therefore to a significant quadrature component. For a quantitative approach, consider the response $u$ of an oscillator (that is, the WG mode) to a given harmonic $\omega_{n}=n \omega_{1}$ of the laser repetition rate

$$
\frac{d^{2} u}{d t^{2}}+\gamma \frac{d u}{d t}+\omega_{0}^{2} u=\exp \left(i \omega_{n} t\right) \cos (\Omega t)
$$

where $\omega_{0}$ and $\Omega$ are the angular frequencies of the oscillator and the pump modulation, respectively, and $\gamma$ is a damping constant. The general steady-state solution is

$$
\begin{aligned}
u= & \frac{\frac{1}{2} \exp \left[i\left(\omega_{n}-\Omega\right) t\right]}{\omega_{0}^{2}+i\left(\omega_{n}-\Omega\right) \gamma-\left(\omega_{n}-\Omega\right)^{2}} \\
& +\frac{\frac{1}{2} \exp \left[i\left(\omega_{n}+\Omega\right) t\right]}{\omega_{0}^{2}+i\left(\omega_{n}+\Omega\right) \gamma-\left(\omega_{n}+\Omega\right)^{2}} .
\end{aligned}
$$

However, $\gamma \ll \omega_{n}$ (low damping) and $\Omega \ll \omega_{n}$ (low modulation frequency) in our case. If, in addition, we assume that the mode is excited very close to resonance, so $\omega_{0}-\omega_{n} \ll \gamma$, we obtain

$$
u \approx \frac{\tau}{2 i \omega_{n}\left(1+\Omega^{2} \tau^{2}\right)} \exp \left(i \omega_{n} t\right)[\cos (\Omega t)+\Omega \tau \sin (\Omega t)],
$$

where $\tau=2 / \gamma$ is the lifetime of the WG mode. The ratio of the quadrature term $\left[u_{Y} \propto \sin (\Omega t)\right]$ to the in-phase term $\left[u_{X}\right.$ $\propto \cos (\Omega t)]$, given by $u_{Y} / u_{X}=\Omega \tau$, confirms that modes with larger $\tau$ are preferentially detected through $u_{Y}$. For a mode very close to resonance, the quality factor $Q=\omega_{0} / \gamma$ of the WG mode is therefore given by

$$
Q=\frac{u_{Y}}{u_{X}} \frac{\omega_{0}}{2 \Omega} .
$$

In practice, however, one should consider $\omega_{0}-\omega_{n} \lesssim \gamma$, a sufficient condition for exciting a WG mode. In this case, one can show that the quantity on the right-hand side of Eq. (4) is equal to $\left\{Q^{-2}+4\left[\left(\omega_{0}-\omega_{n}\right) / \omega_{0}\right]^{2}\right\}^{-1 / 2} \cdot{ }^{36}$ Equation (4) therefore provides a lower bound for $Q$. To find this lower bound for $Q$ in the experiment, it is convenient to average the data for the WG mode in the frequency domain

\section{B. Fourier analysis of the data}

The moduli $|F|$ of the 2D spatial and one-dimensional (1D) temporal Fourier transforms of the wave fields $f(x, y, t)$ for the in-phase and quadrature components are shown as intensity plots in the frequency-wave number $\left(k_{y}\right)$ plane at $k_{x}=0$ in Figs. 2(a) and 2(b), respectively. The frequency step is equal to the repetition rate of the laser $\omega_{1} / 2 \pi=76 \mathrm{MHz}$. In Fig. 2(a), the SAWs propagating in the $-y$ direction dominate the in-phase response, with a phase velocity $3000<v$ $<4000 \mathrm{~ms}^{-1}$. The numerically determined dispersion curve, ${ }^{29-31}$ assuming an average $\mathrm{Cu}$ thickness of $275 \mathrm{~nm}$ (dashed line), shows good agreement. In Fig. 2(b), a WG mode at $760 \mathrm{MHz}$ dominates the quadrature response at both 


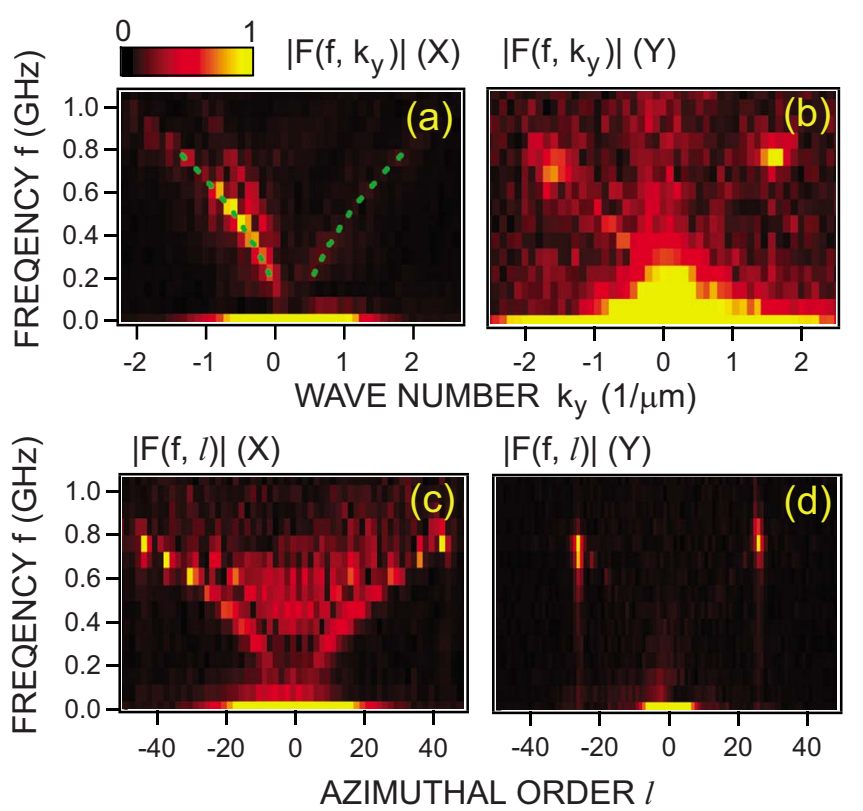

FIG. 2. (Color online) Dispersion relations in the $y$ direction at $k_{x}=0$ for (a) in-phase and (b) quadrature components. Dashed curves in (a) correspond to a numerical simulation. (c) and (d) Dispersion relations from a radial-azimuthal decomposition.

positive and negative $k_{y}$, with $v=3000 \pm 100 \mathrm{~ms}^{-1}$. Within the experimental error, $v$ is equal to that of the SAWs. 2D spatial mapping of the WG mode is best revealed by a temporal Fourier transform $F(\mathbf{r}, f)$, shown for the quadrature component in Figs. 3(a)-3(c) at $f=760 \mathrm{MHz}$ for the modulus, the real part and the phase. The mode is a standing wave, evident in an animation, ${ }^{28}$ with 26 periods visible in $\operatorname{Re}(F)$ and with adjacent half periods differing in $\arg (F)$ by $\pi$.

Fourier analysis in Cartesian coordinates $(x, y)$ thus reveals the wave dispersion. However, a more appropriate Fourier analysis for a WG mode is a radial-azimuthal decomposition from polar coordinates $(r, \theta)$,

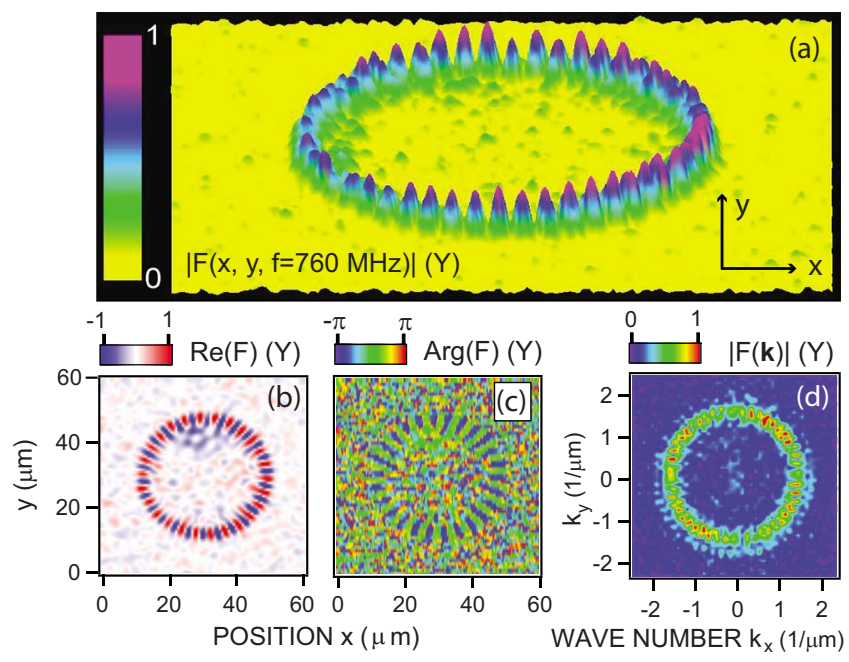

FIG. 3. (Color online) (a) Modulus, (b) real part, and (c) phase of the temporal Fourier transform $F(x, y, f)$ of the quadrature component at $f=760 \mathrm{MHz}$. (d) Corresponding constant-frequency surface of the spatiotemporal Fourier transform $\left|F\left(k_{x}, k_{y}, f\right)\right|$. Image smoothing was applied in (b)-(d).

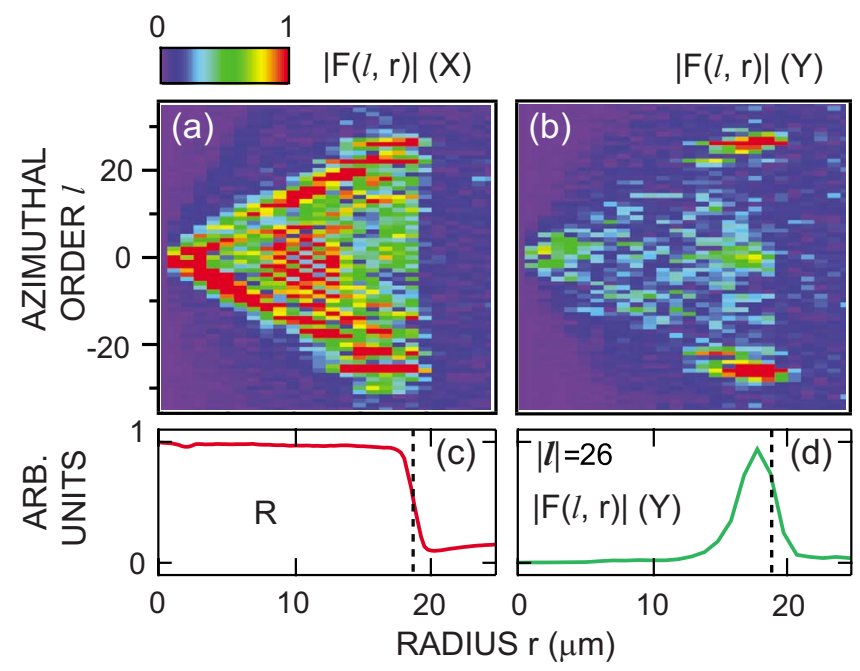

FIG. 4. (Color online) Plots of the modulus of the radialazimuthal Fourier transform $|F(l, r)|$ at $760 \mathrm{MHz}$ for (a) in-phase and (b) quadrature components, respectively. (c) Normalized probe reflectivity $R$ vs radius. (d) Quadrature component of $|F(l, r)|$ vs radius at $760 \mathrm{MHz}$ for $|l|=26$. Vertical dotted lines in (c) and (d) show the disk boundary.

$$
F(r, l, \omega)=\frac{1}{(2 \pi)^{2}} \int_{-\infty}^{\infty} \int_{0}^{2 \pi} f(r, \theta, t) \exp [i(\omega t-l \theta)] d \theta d t,
$$

where $l$ is the azimuthal order (or angular mode number). $|F(r, l, f)|$ is shown as an intensity plot in the $l-r$ plane for both the in-phase and quadrature components at $760 \mathrm{MHz}$ in Figs. 4(a) and 4(b), respectively, where $l>0$ corresponds to anticlockwise wave motion. For the in-phase case, SAWs contribute over a triangular area up to $r \approx a$, where $a$ $=18.75 \mu \mathrm{m}$ is the disk radius. A similar shape results from an expansion using Eq. (5) of a monochromatic plane wave propagating in the $-y$ direction. The detection sensitivity falls for $r>a$ due to the decrease in the probe reflectivity, as shown in Figs. 1(b) and 4(c). For the quadrature case in Fig. 4(b), the WG mode yields equal maxima centered at $l$ $= \pm 26$ and $r=18 \mu \mathrm{m}$. An average section of this plot for $l$ $= \pm 26$ is shown in Fig. 4(d). The WG mode is confined within $\sim 4 \mu \mathrm{m}$ of the disk radius, a distance of the order of the acoustic wavelength $\Lambda=(2 \pi a) / 26 \approx 4.5 \mu \mathrm{m}$. This localization distance also depends on the sound velocities of the materials inside and outside the waveguide. ${ }^{32}$ Data for $|F(r, l, f)|$ at $r=18 \mu \mathrm{m}$ are shown in Figs. 2(c) and 2(d) for the in-phase and quadrature components, respectively, and are consistent with the results for the $y$ direction. ${ }^{37}$ Figure 3(d) shows a $760 \mathrm{MHz}$ constant-frequency surface in $\mathbf{k}$ space for the spatiotemporal Fourier transform $|F(\mathbf{k})|$. Interestingly, 52 petals (determined by $2 l$ ), as seen for $|F(\mathbf{r})|$ in Fig. 3(a), again appear. This angular periodicity in $|F(\mathbf{k})|$ and $|F(\mathbf{r})|$ only arises for standing WG modes, as can be shown by Fourier analysis of $f(\mathbf{r}, t)=g(r) \cos \left(l \theta \pm \omega_{0} t\right)$ and $g(r) \cos (l \theta) \cos \left(\omega_{0} t\right)$

The radial-azimuthal decomposition in Figs. 4(a) and 4(b) is suited for finding a lower bound for the WG mode $Q$ 
factor using Eq. (4) (where $u \equiv|F|$ ). An average over $l$ $= \pm 26$ gives $u_{Y} / u_{X} \approx 1.6$ and, from Eq. (4), $Q \gtrsim 600$. Given the occurrence of $Q / \pi$ periods of a damped oscillator before decay to $1 / e$ of the initial amplitude, this corresponds to $\gtrsim 7$ circuits around the disk (similar to Rayleigh's WG modes in St. Paul's!), a distance of $\gtrsim 800 \mu \mathrm{m}$, or to a lifetime $\tau$ $\gtrsim 0.25 \mu \mathrm{s}$ (all corresponding to lower bounds). The ultrasonic attenuation coefficient ${ }^{33}$ of bulk longitudinal waves in the underlying (relatively lossy) $\mathrm{SiO}_{2}$ at $760 \mathrm{MHz}$, $\sim 100 \mathrm{~mm}^{-1}$, can explain this, although the other films and curvature radiation losses will also contribute. A comparable value of $\tau$ was found at $460 \mathrm{MHz}$ for a microscopic ring resonator in a similar film stack. ${ }^{34}$ The derived lower bound for $Q$ is not sufficiently low to explain the smearing of the WG mode frequency in Fig. 2(d) by $\sim \pm 80 \mathrm{MHz}$. This may be caused by variations in the detection sensitivity as a function of delay time. Similar effects as a function of position can account for the spread in $l$ in Fig. 4(b).

Other WG modes for our range of excited frequencies up to $\sim 1 \mathrm{GHz}$ are notably absent. Since the $l$ th mode frequency is $f=v(l) l /(2 \pi a)$, the mode separation for $l=26$ is $v(l) /(2 \pi a) \approx 25 \mathrm{MHz}$. The resonance width $\sim \omega_{0} /(2 \pi Q)$ $\gtrsim 1 \mathrm{MHz}$ is small in comparison, so the condition for overlap with the $n$th harmonic (here $n=10$ ) of the laser repetition rate $76 \mathrm{MHz}$ is relatively stringent. The chance of an overlap increases with increasing $a$.

\section{DISCUSSION AND CONCLUSIONS}

A key question arises as to how the WG mode is guided. A larger velocity outside the disk is one necessary condition for the existence of "true" SAW WG modes. True WG modes result from surface curvature ${ }^{2}$ and do not exist at an equivalent linear boundary. We should therefore first consider the little-studied problem of SAWs interacting with a linear boundary between two different thin films on a substrate. Guided waves of this kind have not been studied theoretically, nor has the simpler problem of such waves at the boundary between two solid "quarter-spaces." 35 An interface wave may also exist if one of the media is inhomogeneous near the interface. ${ }^{32}$ Indeed, experiments on straight edges with the same dished structure as in the present investigation did reveal guided modes with a velocity slightly less than that of the SAWs in the surrounding $\mathrm{Cu},{ }^{31}$ which leads us to conclude that we are detecting a WG-like mode associated with the $\mathrm{Cu}$ film thickness nonuniformity. The mode is guided because the thicker copper near the edge provides a waveguide channel with a lower SAW velocity compared to both the interior of the disk and the silica layer outside. In fact, separate Fourier analysis of the imaged region outside the disk corresponding to the silica layer gives a SAW velocity $\sim 30 \%$ larger than in the disk interior.

This work should be followed up with experiments on flat surfaces and on different materials to establish general criteria for the existence of WG modes in systems of thin films on substrates. It would also be interesting to investigate the optimal conditions for coupling the excitation to the WG mode(s) by the choice of spot position and spot shape.

In conclusion, we have dynamically imaged coherent surface phonons confined in a whispering-gallery-like mode in a microscopic disk. By modulating the optical pump beam, we extract a quadrature component that allows the mode spatial distribution in real space and in $\mathbf{k}$ space to be obtained by Fourier analysis and allows the $Q$ factor and mode lifetime to be estimated from a radial-azimuthal decomposition. In future, by tuning the laser repetition rate to the $\mathrm{WG}$ resonance, precise values for $Q$ will be obtainable. This approach should open the way to the detailed study of the form and lifetime of confined sound fields in more complicated geometries such as two coupled disks, chains of disks, chaotic cavities, or phononic crystal resonators, also providing useful analogies with electromagnetic or quantum-mechanical systems where analytical modeling is impossible. Furthermore, our technique should be applicable to hybrid microscopic resonators, such as microdisc lasers, which can simultaneously confine optical and acoustic waves.

\footnotetext{
*Corresponding author; assp@ kino-ap.eng.hokudai.ac.jp

${ }^{1}$ Lord Rayleigh, Philos. Mag. 20, 1001 (1910).

${ }^{2}$ L. M. Brekhovskikh, Sov. Phys. Acoust. 13, 462 (1968).

${ }^{3}$ S. Tamura, Phys. Rev. B 79, 054302 (2009).

${ }^{4}$ J. R. Wait, Can. J. Phys. 45, 1861 (1967).

${ }^{5}$ J. W. Dickey, G. V. Frisk, and H. Uberall, J. Acoust. Soc. Am. 59, 1339 (1976).

${ }^{6}$ W. Hassan and P. B. Nagy, J. Acoust. Soc. Am. 101, 2496 (1997).

${ }^{7}$ D. Clorennec and D. Royer, Appl. Phys. Lett. 82, 4608 (2003).

${ }^{8}$ Y. Fan, F. Honarvar, A. N. Sinclair, and M.-R. Jafari, J. Acoust. Soc. Am. 113, 102 (2003)

${ }^{9}$ S. Ishikawa, N. Nakaso, N. Takeda, T. Mihara, Y. Tsukahara, and K. Yamanaka, Appl. Phys. Lett. 83, 4649 (2003).

${ }^{10}$ J. R. Clark, W.-T. Hsu, M. A. Abdelmonneum, and C. T.-C. Nguyen, J. Microelectromech. Syst. 14, 1298 (2005).

${ }^{11}$ M. Trigo, A. Bruchhausen, A. Fainstein, B. Jusserand, and
}

V. Thierry-Mieg, Phys. Rev. Lett. 89, 227402 (2002).

${ }^{12}$ T. J. Kippenberg and K. J. Vahala, Science 321, 1172 (2008).

${ }^{13}$ M. Tomes and T. Carmon, Phys. Rev. Lett. 102, 113601 (2009).

${ }^{14}$ K. J. Vahala, Nature (London) 424, 839 (2003).

${ }^{15}$ I. S. Grudinin, V. S. Ilchenko, and L. Maleki, Phys. Rev. A 74, 063806 (2006)

${ }^{16}$ A. M. Armani, R. P. Kulkarni, S. E. Fraser, R. C. Flagan, and K. J. Vahala, Science 317, 783 (2007).

${ }^{17}$ G. Fasching, V. Tamosiunas, A. Benz, A. M. Andrews, K. Unterrainer, R. Zobl, T. Roch, W. Schrenk, and G. Strasser, IEEE J. Quantum Electron. 43, 687 (2007).

${ }^{18} \mathrm{~S}$. Bittner, B. Dietz, M. Miski-Oglu, P. O. Iriarte, A. Richter, and F. Schafer, Phys. Rev. A 80, 023825 (2009).

${ }^{19}$ R. M. Cole, Y. Sugawara, J. J. Baumberg, S. Mahajan, M. Abdelsalam, and P. N. Bartlett, Phys. Rev. Lett. 97, 137401 (2006).

${ }^{20}$ B. Min, E. Ostby, V. Sorger, E. Ulin-Avila, L. Yang, X. Zhang, and K. Vahala, Nature (London) 457, 455 (2009). 
${ }^{21}$ L. Sun, Z. Chen, Q. Ren, K. Yu, L. Bai, W. Zhou, H. Xiong, Z. Q. Zhu, and X. Shen, Phys. Rev. Lett. 100, 156403 (2008).

${ }^{22}$ H. D. Dardy, L. Flax, C. F. Gaumond, J. V. Subrahmanyam, S. Ashrafi, P. K. Raju, and H. Uberall, J. Acoust. Soc. Am. 82, 1378 (1987).

${ }^{23}$ M. L. M. Balistreri, D. J. W. Klunder, F. C. Blom, A. Driessen, H. W. J. M. Hoekstra, J. P. Korterik, L. Kuipers, and N. F. van Hulst, Opt. Lett. 24, 1829 (1999).

${ }^{24}$ H. Gersen, D. J. W. Klunder, J. P. Korterik, A. Driessen, N. F. van Hulst, and K. Kuilers, Opt. Lett. 29, 1291 (2004).

${ }^{25}$ Y. Sugawara, O. B. Wright, O. Matsuda, M. Takigahira, Y. Tanaka, S. Tamura, and V. E. Gusev, Phys. Rev. Lett. 88, 185504 (2002).

${ }^{26}$ T. Tachizaki, T. Muroya, O. Matsuda, O. B. Wright, Y. Sugawara, and D. H. Hurley, Rev. Sci. Instrum. 77, 043713 (2006).

${ }^{27}$ R. E. Vines, M. R. Hauser, and J. P. Wolfe, Z. Phys. B 98, 255 (1995).

${ }^{28}$ See supplementary material at http://link.aps.org/supplemental/ 10.1103/PhysRevB.81.165434 for animations.

${ }^{29}$ J. A. Rogers, A. A. Maznev, M. J. Banet, and K. A. Nelson, Annu. Rev. Mater. Sci. 30, 117 (2000).
${ }^{30}$ G. Farnell and E. L. Adler, in Physical Acoustics, edited by W. P. Mason and R. N. Thurston (Academic, New York, 1972), Vol. 9 , p. 35 .

${ }^{31}$ See A. A. Maznev, T. A. Kelf, M. Tomoda, O. Matsuda, and O. B. Wright, J. Appl. Phys. 107, 033521 (2010). This paper gives the densities and elastic constants of $\mathrm{Cu}, \mathrm{Ta}, \mathrm{Si}_{3} \mathrm{~N}_{4}, \mathrm{SiO}_{2}$, and Si. A higher-order (Sezawa) SAW mode is also visible in Fig. 2(a).

${ }^{32}$ A. A. Oliner, in Surface Acoustic Waves, edited by A. A. Oliner (Springer, Berlin, 1978), p. 187.

${ }^{33}$ T. C. Zhu, H. J. Maris, and J. Tauc, Phys. Rev. B 44, 4281 (1991).

${ }^{34}$ A. A. Maznev, Ultrasonics 49, 1 (2009).

${ }^{35}$ A. P. Mayer, Ultrasonics 48, 478 (2008).

${ }^{36}$ This can be shown by considering the low-frequency response of the photodiode (at angular frequency $\Omega \ll \omega_{1}$ ) that collects the reflected train of probe light pulses at frequency $\omega_{1}$. The approximation $\omega_{0} \approx \omega_{n}$ was also made here.

${ }^{37}$ The quadrature data agree if $\left(2 \pi a_{M}\right) / l=2 \pi / k_{y}$ or $k_{y}=l / a_{M}$, where $a_{M}=17 \pm 1 \mu \mathrm{m}$. 